\title{
Partial Differential Equations of A Virus Dynamical Model
}

\author{
Urbain Traoré \\ Laboratory of Mathematics and Informatique (LAMI), Joseph KI-ZERBO University, Ouagadougou, Burkina Faso.
}

How to cite this paper: Urbain Traoré. (2021) Partial Differential Equations of A Virus Dynamical Model. Journal of Applied Mathematics and Computation, 5(4), 321-330.

DOI: 10.26855/jamc.2021.12.010

Received: November 1, 2021

Accepted: November 25, 2021

Published: December 13, 2021

*Corresponding author: Urbain Traoré, Laboratory of Mathematics and Informatique (LAMI), Joseph KI-ZERBO University, Ouagadougou, Burkina Faso.

Email: urbain.traore@yahoo.com

\begin{abstract}
In this work, we investiguate a reaction-diffusion model with homogeneous Neumann boundary conditions of virus transmission. The model that we study in work is a generalization of the model developed by Zhang, et al.; in other words, we are trying to understand the influence of diffusion on virus dynamics. We prove global existence, uniqueness, positivity and boundness of the solution using a variational theory and some other useful tools from functional analysis. From the characteristic equation, we derive the local stability of the equilibriums. Moreover, the global asymptotical properties of the free-virus equilibrium and the endemic equilibruims of the model are studied by constructsing of a suitable Lyapunov functions. Finally, numerical simulations are performed to support the theoretical results obtained. Our numerical results indicate that the introduction of the diffusion in the model does not suppressthe dynamics of the virus, one obtains globally the configurations that in the absence of thediffusion. However, the mathematical analysis becomes more complicated.
\end{abstract}

\section{Keywords}

Cell-to-cell transmission, Stability, Reaction-diffusion virus, Model

\section{Introduction}

Viral diseases have been known for millennia. Already under the Babylonians, it was known that rabies is transmitted by the bite of the rabid dog. Smallpox, a disease causing high mortality, has accompanied humans foralong time and has been found on mummies in Egypt.

In 1884, the development of the Chamberland Candles [1], which eliminated bacteria from a solution, represented the first step in the discoveryof viruses. Adolf Mayer (1843-1942) described in detail a disease of tobacco plants which he called tobacco mosaic. He realizes that the disease isinfectious because it can be transmitted by what he believes to be bacteria.

The tobacco mosaic virus (TMV/VMT) will remain an importantmodel in all fundamental virus studies. In 1935, Wendell Stanley succeeded in crystallizing the tobacco mosaic virus (TMV/VMT), which allowed its chemicalanalysis and the following year Bawden and Pirie described astructure combining proteins and ribonucleic acid. The first human virus,the agent of yellow fever, was identified in 1901 by Walter Reed, James Carroll and Jesse Lazear. Another example is the Ebola virus, which causes Ebola hemorrhagic fever with a mortality rate from $50 \%$ to $90 \%$.

Currently, new viruses continue to be discovered, such as the hepatitis C virus in 1989, the Nipah virus in 1999 (respiratory infection in pigs andencephalitis in humans), Metapneumovirus in 2001, the SARS virus (severeacute respiratory syndrome) in 2003.

There exists in the literature, several mathematical models using ordinary differential equations (ODEs), delay 
differential equations (DDEs) and partial differential equations (PDEs), have been developed to help understand the dynamics the mechanisms and dynamics of within-host viralinfections (see [2, 3, 4, 5, 6]).

There have been many advances in the understanding of infections ofcells by viruses, one can quote on this subject the work of Spouge and al forthe transmission of virus to cell to cell via virological synapses ( $\mathrm{cf}$ [10]). In Zhang et al. [11], they have developed the following model of viral transmissionbased on ODE with two different types of infection and a cure rate:

$$
\text { (1) }\left\{\begin{array}{l}
\frac{\partial H}{\partial t}=\Lambda-d H-(\beta I+\alpha V) H+\rho I \\
\frac{\partial I}{\partial t}=(\beta I+\alpha V) H-(a+\rho) I \\
\frac{\partial V}{\partial t}=k I-u V
\end{array}\right.
$$

where $H ; I$ and $V$ represent the concentrations of uninfected cells,infected cells, and free virus respectively. $\pi$ is the regeneration rate of uninfected cells, $d ; a$ and $u$ are the death rates of three kinds of cellsrepresentsthe curate, $k I$ is the rate at which infected cells produce free viruses. $(\beta I+\alpha V) H$ represents the total infection rate of host cells, which is divided into two parts $\beta I H$ and $\alpha V H$. The former represents the part whereinfected cells infect host cells by direct contact, and the lattermeans that host cells are infected by the free virus. Using a Lyapunov functions, the authors have establihed the local andglobal stability of the points of equilibrium.

In the basic mathematical model proposed by Zhang et al. [11], as well as in the other models which resulted fromthis work (cf [12]), thespatial mobility of cells and viruses is ignored. Taking spatial movementinto account inmany biological phenomena is no longer to be justifed, asevidenced by the work of Funk et al. [13] on the study of virus-immunespatial dynamics, reaction-diffusion models of the hepatitis B (see [14]). Motivated by the work of Zhang et al., we introduce diffusion into their model and analyze the impact ofspatial mobility on equilibrium states. More precisely, we are interested in the following model:

$$
\text { (2) }\left\{\begin{array}{l}
\frac{\partial H}{\partial t}=\Lambda-d H-(\beta I+\alpha V) H+\rho I \\
\frac{\partial I}{\partial t}=(\beta I+\alpha V) H-(a+\rho) I \\
\frac{\partial V}{\partial t}=d_{V} \Delta V+k I-u V
\end{array}\right.
$$

with homogeneous Neumann boundary conditions

$$
\text { (3) } \frac{\partial V}{\partial \eta}=0 \text { on } \Omega \times(0, \infty)
$$

and initial conditions

$$
\text { (4) } H(x, 0)=H_{0}, \quad I(x, 0)=I_{0}, \quad V(x, 0)=V_{0} x \in \Omega \text {. }
$$

Here $\Omega$ is a bounded domain in $\mathbb{R}^{n}$ with smooth boundary $\partial \Omega, \frac{\partial V}{\partial \eta}$ is the normal derivative of $V$ on $\partial \Omega$.

The rest of paper is organized as follows. The next section deals with theglobal existence, positivity, and boundedness of solutions of problem (2)-(4). In Section 3, we discuss the stability analysis of equilibria. In Section 4, we present the numerical simulation to illustrate our result.

\section{Global existence, positivity, and boundedness of solution}

This section is devoted to the study of the global existence, positivity, and boundedness of solutions of problem 2-4. We state the following result.

Theorem 1. For any given initial data $\Phi=\left(H_{0}, I_{0}, V_{0}\right)^{T}$ satisfying condition (4); there exists a unique solution of system (2) defined on $[0,+\infty)$ and this solution remains nonnegative and bounded for all $t \geq 0$.

Proof. We define the Banach space $X=(C(\bar{\Omega}))^{3}$; problem can be written abstractly in the space $X$ as follow: 
(5) $\left\{\begin{array}{c}u^{\prime}(t)=A u(t)+F(u(t)), \quad t>0 \\ u(0)=u_{0} \in C(\Omega)\end{array}\right.$

where $u=(H, I, V)^{T}, \quad u_{0}=\left(H_{0}, I_{0}, V_{0}\right)^{T}$ and $A u=\left(0,0, d_{V} \Delta u\right)^{T}$ and

$$
F(u(t))=\left(\begin{array}{c}
\Lambda-d H-(\beta I+\alpha V) H+\rho I \\
(\beta I+\alpha V) H-(a+\rho) I \\
k I-u V
\end{array}\right)
$$

Since $F$ is locally Lipschitz in $X$, thus there exists a unique local solution of the system (2) on the interval $\left[0, T_{\max }\right)$; where $T_{\max }$ denotes the maximal existence time for solution of the system (2) (see [15]).

We have $H(x, t) \geq 0, I(x, t) \geq 0, V(x, t) \geq 0$, since $\mathbf{0}=(0,0,0)$ represents a lower-solution of the model (5).

In order to prove the boundedness of solutions, let us set $W=H+I$. This implies

$$
\begin{aligned}
\frac{\partial W}{\partial t} & =\Lambda-d H-\rho I-(a+\rho) I \\
& =\Lambda-d H-a I \\
& =\Lambda-\gamma_{1} W
\end{aligned}
$$

where $\gamma_{1}=\min (i d, a)$. Hence

$$
W(x, t) \leq \max \left\{\frac{\Lambda}{\gamma_{1}}, \max _{x \in \bar{\Omega}}\left(H_{0}(x)+I_{0}(x)\right)\right\}
$$

This implies that $H$ and $I$ are bounded. Furthermore, from the boundness for $I$ and the system (2); it follows that $V$ satisfies the following system

$$
\begin{aligned}
\frac{\partial V}{\partial t}-d_{V} \Delta V & \leq k \gamma_{2}-u V \quad \text { in } \Omega *\left[0, \quad T_{\max }\right) \\
\frac{\partial V}{\partial \eta} & =0 \quad \text { in } \partial \Omega *\left[0, \quad T_{\max }\right) \\
V(x, 0) & =V_{0}(x) \text { on } \Omega
\end{aligned}
$$

where $\gamma_{2}=\max \left\{\frac{\Lambda}{\gamma_{1}}, \max _{x \in \bar{\Omega}}\left(H_{0}(x)+I_{0}(x)\right)\right\}$.

Using the comparison principle [16], we deduce that $V \leq \tilde{V}$; where $\tilde{V}(t)=V_{0}(x) e^{-u t}+\frac{k \gamma_{2}}{u}\left(1-e^{-u t}\right)$ is the solution fo the following ODE

$$
\begin{gathered}
\frac{\partial \widetilde{V}}{\partial t}=k \gamma_{2}-u \widetilde{V} \quad \text { on } \quad\left[0, T_{\max }\right) \\
\widetilde{V}(0)=\left\|V_{0}\right\|_{\infty}=\max _{x \in \bar{\Omega}}\left(V_{0}(x)\right) .
\end{gathered}
$$

Since $\tilde{V} \leq \max \left\{\frac{k \gamma_{2}}{u},\left\|V_{0}\right\|_{\infty}\right\}$ for all $t \in(0, \infty)$; we have that

$$
\text { (8) } \quad V(x, t) \leq \max \left\{\frac{k \gamma_{2}}{u},\left\|V_{0}\right\|_{\infty}\right\} \text { for all }(x, t) \epsilon \bar{\Omega} X\left[0, T_{\max }\right) \text {. }
$$

Using [17], Theorem 2, to establish the $L^{\infty}$ uniform boundedness of $V$, it is sufficient to show the $L^{1}$ uniform boundedness.

Since $\frac{\partial V}{\partial \eta}=0$ and

$$
\frac{\partial(H+I+V)}{\partial t}-\Delta\left(d_{V} V\right) \leq \Lambda+k \gamma_{2}-\gamma_{3}(H+I+V),
$$

where $\gamma_{3}=\min \left\{\gamma_{1}, \gamma_{2}\right\}$, we get

$$
\frac{\partial}{\partial t}\left(\int(H+I+V) d x\right) \leq \operatorname{meas}(\Omega)\left(\pi+k \gamma_{2}\right)-\gamma_{3}\left(\int(H+I+V) d x\right) .
$$

Hence 


$$
\int(\mathrm{H}+\mathrm{I}+\mathrm{V}) \mathrm{dx} \leq \operatorname{meas}(\Omega)\left(\max \left\{\frac{\Lambda+\mathrm{k} \gamma_{2}}{\gamma_{3}}, \quad\left\|\mathrm{H}_{0}+\mathrm{I}_{0}+\mathrm{V}_{0}\right\|_{\infty}\right\}\right)
$$

which implies that

$$
\operatorname{Sup}_{t \geq 0}\left(\int V(x, t) d x\right) \leq K:=\operatorname{meas}(\Omega)\left(\max \left\{\frac{\Lambda+k \gamma_{2}}{\gamma_{3}},\left\|H_{0}+I_{0}+V_{0}\right\|_{\infty}\right\}\right)
$$

Using [17, Theorem 3.1], we deduce that there exists a positive constant $K^{*}$ that depends on $K$ and on $\| H_{0}+I_{0}+$ VO//o such that

$$
\operatorname{Sup}_{t \geq 0}\|V(., t)\|_{\infty} \leq K^{*}
$$

From the above, we have proved that $H, I$ and $V$ are bounded on $\bar{\Omega} X\left[0, T_{\max }\right)$. Therefore, it follows from the standard theory for semilinear parabolic systems (see [18]) that $T_{\max }=+\infty$. This completes the proof of the theorem.

\section{Qualitative analysis of the spacial model}

The basis reproduction number $[19,20]$ of the model (2); is given by

$$
R_{0}=\frac{\Lambda(\alpha k+\beta u)}{d u(a+\rho)}
$$

It is not hard to show that the system (2); is always a disease-free equilibrium of the form $E_{0}=\left(h_{0}, 0,0\right)$, where $h_{0}=\frac{\Lambda}{\mathrm{d}}$. Further, if $R_{0}>1$; the system (2); has a unique endemic equilibrium $E^{*}=\left(H^{*}, I^{*}, V^{*}\right)$ where

$$
\begin{gathered}
H^{*}=\frac{\Lambda}{d R_{0}} \\
I^{*}=\frac{\Lambda}{a}\left(1-\frac{1}{R_{0}}\right) \\
\text { (15) } V^{*}=\frac{k}{u} I^{*} .
\end{gathered}
$$

The objective of this section is to discuss the local and global stability of the equilibria.

\subsection{Local stability of the equilibria}

We now discuss the stability of the corresponding steady states of the model. Let us assume that $0<\lambda_{1}<\lambda_{2}<\cdots<$ $\lambda_{n}$ be the eigenvalues of the operator $-\Delta$ on $\Omega$ with the homogeneous Neumann boundary conditions and $\varepsilon\left(\lambda_{i}\right)$ be the eigenfunction space corresponding to $\lambda_{i}$ in $C^{1}(\Omega)$. Let $\left\{\psi_{i j}: j=1,2 \ldots, \operatorname{dim} \varepsilon\left(\lambda_{i}\right)\right\}$ represent an orthonormal basis of $\varepsilon\left(\lambda_{i}\right), \mathbb{x}_{i j}=\left\{\phi \psi_{i j}: \phi \in \mathbb{R}^{3}\right\}$ and $\mathbb{x}=\left(C(\Omega)^{1}\right)^{3}$, then

$$
\mathbb{x}=\bigoplus_{i=1}^{\infty} \mathbb{x}_{i} \quad \text { and } \quad \mathbb{x}_{i}=\bigoplus_{j=1}^{\operatorname{dimE}\left(\lambda_{i}\right)} \mathbb{x}_{i j} .
$$

Let $\bar{E}=(\bar{H}, \bar{I}, \bar{V})$ be an arbitrary steady state of the system (2). We define the following perturbation about $\bar{E}$ :

$$
U_{1}(x, t)=H(x, t)-\bar{H}, U_{2}(x, t)=I(x, t)-\bar{I} \text { and } U_{3}(x, t)=V(x, t)-\bar{V}
$$

Linearizing the system (2); at $\overline{\boldsymbol{E}}$; we obtain

$$
\text { (17) } \frac{\partial U}{\partial t}=D \Delta U+J * U(x, t)
$$

where

$$
J=\left(\begin{array}{ccc}
-d-(\beta \bar{I}+\alpha \bar{V}) & \rho-\beta \bar{H} & \alpha \bar{H} \\
(\beta \bar{I}+\alpha \bar{V}) & \beta \bar{H}-(a+\rho) & \alpha \bar{H} \\
0 & k & -u
\end{array}\right)
$$

is the Jacobian matrix of (16); without diffusion ( i.e. $\left.d_{v}\right)$ at $\bar{E}$ and $D=\operatorname{diag}\left(0,0, d_{v}\right)$ and $U=\left(U_{1}, U_{2}, U_{3}\right)^{T}$. 
We define the operator $\boldsymbol{L}$ by

$$
L U=D \Delta U+J * U(x, t)
$$

$\mathbb{x}_{i}$ is invariant under the operator $L$ for all $i \geq 1$. Further, $\eta$ is an eigenvalue of $\boldsymbol{L}$ if and only if it is a root of the equation

$$
\operatorname{det}\left(-\lambda_{i} D+J-I_{3}\right)=0
$$

for some $i \geq 1$ and in this case, there exists an eigenvector in $\gamma_{i} \mathrm{i}$ :

We have

$$
-\lambda_{i} D+J-\eta I_{3}=\left(\begin{array}{ccc}
-d-(\beta \bar{I}+\alpha \bar{V})-\eta & \rho-\beta \bar{H} & \alpha \bar{H} \\
(\beta \bar{I}+\alpha \bar{V}) & \beta \bar{H}-(a+\rho)-\eta & \alpha \bar{H} \\
0 & k & -\lambda_{i} d_{V}-u-\eta
\end{array}\right)
$$

At the boundary equilibrium $E_{0}=\left(h_{0}, 0,0\right)$; it is easy to see that $\eta_{1}=-u-\lambda_{\boldsymbol{i}} d_{V}$ is one root of (18); and the other two roots $\eta_{2}, \eta_{3}$ satisfy

$$
\begin{aligned}
\eta_{2}+\eta_{3} & =-\left(a+\rho+u+\lambda_{i} d_{V}-h_{0}\right) \\
& =\left(\frac{\alpha k}{u R_{0}}+\beta h_{0}\left(\frac{1}{R_{0}}-1\right)+u+\lambda_{i} d_{V}\right) \\
\eta_{2} \eta_{3} & =\left(a+\rho-\beta h_{0}\right)\left(u+\lambda_{i} d_{V}\right)-\operatorname{ak} h_{0} \\
& \geq(a+\rho)\left(u+\lambda_{i} d_{V}\right)\left(1-R_{0}\right)
\end{aligned}
$$

Then, when $R_{0}<1, \eta_{2}+\eta_{3}<0$ and $\eta_{2} \eta_{3}>0$ all the root of (18); at $E_{0}$ have negative real parts then $E_{0}$ is locally asymptotically stable. For $R_{0}>0$; we take $\lambda_{i}=\lambda_{0}=0$ to obtain $\eta_{2} \eta_{3}<0$. Which implies that (18) has a positive eigenvalue and $E_{0}$ is unstable.

Evaluating (18) at $E^{*}$ and using [11], we obtain

$$
A \eta^{3}+B \eta^{2}+C \eta+D=0
$$

where

$$
\begin{gathered}
A=\left(u+\lambda_{i} d_{V}\right) H^{*} \\
B=\rho \Lambda I^{*}+\left(u+\lambda_{i} d_{V}\right)^{2} H^{*}+\alpha k H^{*} \\
C=\alpha \Lambda H^{*}+\alpha u^{2}+\alpha \rho k H^{*} I^{*}+\rho\left(u+\lambda_{i} d_{V}\right)^{2} I^{*}-u a \rho\left(u+\lambda_{i} d_{V}\right) I^{*}-\rho^{2}\left(u+\lambda_{i} d_{V}\right) I^{*}+a \beta\left(u+\lambda_{i} d_{V}\right) I^{*} H^{*} \\
+u \rho \beta I^{*} H^{*},
\end{gathered}
$$

We have, $A, B>0$ and noticing that $H^{*}(u \beta+\alpha k)=u(a+\rho)$ we deduce that

$$
\begin{gathered}
C=\gamma \alpha k H^{*}+\gamma u^{2}+\rho u^{2} I^{*}+u a \beta I^{*} H^{*}>0 \\
D=a \alpha u k I^{*} H^{*}+a \beta u^{2} I^{*} H^{*}>0
\end{gathered}
$$

and

$$
\begin{gathered}
\mathrm{BC}-\mathrm{AD}=\left(\gamma u+\rho u I^{*}+\alpha k H^{* 2}\right) \cdot\left(\alpha \Lambda k H^{*}+u \alpha \beta I^{*} H^{*}+\Lambda u^{2}+\rho u^{2} H^{*}\right) \\
+u^{4} H^{*}\left(\Lambda+\rho I^{*}\right)+\alpha k u^{2} H^{* 2}\left(\Lambda-a I^{*}\right)>0
\end{gathered}
$$

where $R_{0}>1$ and $H^{*}=\Lambda / a\left(1-1 / R_{0}\right)$ are used.

Then, by the Routh-Hurwitz Criterion [21], we know that all the roots of (18) at $E^{*}$ always have negative real parts. Thus, the epidemic equilibrium $E^{*}$ is locally asymptotically stable for $R_{0}>1$. The characterization of the local stability of virus-free equilibrium $E_{0}$ and endemic equilibrium are given by the following result.

Theorem 2. For model (2); we have the following conclusion:

I. $\quad E_{0}$ is locally stable if $R_{0}<1$ and unstable if $R_{0}>1$.

II. $\quad E^{*}$ is locally stable if $R_{0}>1$. 


\subsection{Global stability of the two equilibria}

Our aim in step is establish the global stability of virus-free equilibrium and endemic equilibrium for the for reaction diffusion equations (2) - (4). Inspired by the work of Zhang et al., we construct the Lyapunov functions which allow us under certain conditions to have the global stability of the equilibruim points.

\section{Theorem 3.}

If $R_{0}<1$; the virus-free equilibrium $E_{0}$ is globally asymptotically stable.

Proof. In the case where the mobility of viruses is not taken into account, in the works of Zhang et al., the authors used the following Lyapunovfunctional for the study of the global stability $E_{0}$.

$$
L_{1}(H, I, V)=H-h_{0}-H_{0} \ln \left(\frac{H}{h_{0}}\right)+\frac{\rho}{2(d+a) h_{0}}\left(H-h_{0}+I\right)^{2}+I+p V
$$

with $p>0$ is a constant to be determined.

We introduce the following Lyapunov function

$$
K_{1}(H, I, V)=\int L_{1}(H(x, t), I(x, t), V(x, t)) d x .
$$

Differentiating $K_{1}$ with respect to $t$ along the solutions of the model (2) - (4); we obtain

$$
\frac{d K_{1}}{d t}=-\left(\frac{h_{0}}{H}+\frac{\rho}{H h_{0}}+\frac{d \rho}{(d+a) h_{0}}\right)\left(H-H_{0}\right)^{2}+k\left(p-\frac{a+\rho+\beta h_{0}}{k}\right) I+\left(\frac{\alpha h_{0}}{u}-p\right) u v+\mathrm{p} d_{V} \int \Delta V d x
$$

Combining the divergence theorem and homogeneous Neumann boundary conditions(4); we have

$\int \Delta V d x=0$ and since $R_{0}<1$; we have $(\alpha u+\beta k)<u(a+\rho)$; then taking $p>0$ such that $\frac{\beta h_{0}}{u}<p<\frac{\left(a+\rho-\alpha h_{0}\right)}{k}$. It follows that $\frac{d K_{1}}{d t}<0$. Consequently, $E_{0}$ is globally asymptoticallystable.

\section{Theorem 4.}

If $1<R_{0} \leq 1+\delta$; the epidemic equilibrium $E^{*}$ is globally asymptotically stable, where

$$
\delta=\frac{\left(\beta \pi+(a-\rho) d+\sqrt{(\beta \pi+(a-\rho) d)^{2}+4 a \rho d^{2}}\right)}{2 \rho d} .
$$

Proof.

Assuming that $R_{0}>1$; we define a Lyapunov functional for the system (2) - (4); at $E^{*}$ as follows

$$
K_{2}(H, I, V)=\int L_{2}(H(x, t), I(x, t), V(x, t)) d x
$$

where

$$
\begin{aligned}
L_{2}(H, I, V)= & H-H^{*}-H^{*} \ln \left(\frac{H}{H^{*}}\right)+\left(I-I^{*}-I^{*} \ln \left(\frac{I}{I^{*}}\right)\right) \\
& +\frac{\alpha H^{*} V^{*}}{k I^{*}}\left(V-V^{*}-V^{*} \ln \left(\frac{V}{V^{*}}\right)\right) \\
& +\frac{\rho}{2(d+a)}\left(H-H^{*}+I-I^{*}\right)^{2}
\end{aligned}
$$

Differentiating $K_{2}$ with respect to $t$ along the solutions of the model (2) - (4); we obtain

$$
\begin{aligned}
\frac{\partial K_{2}}{\partial t} & =H-H^{*}-H^{*} \ln \left(\frac{H}{H^{*}}\right)+\left(I-I^{*}-I^{*} \ln \left(\frac{I}{I^{*}}\right)\right) \\
& =\left(H-H^{*}-H^{*} \ln \left(\frac{H}{H^{*}}\right)\right) \frac{\alpha H^{*} V^{*}}{k I^{*}} \\
& =\frac{\rho}{2(d+a)}\left(H-H^{*}+I-I^{*}\right)^{2} .
\end{aligned}
$$

Differentiating $K_{2}$ with respect to $t$ along the solutions of the model (2) - (4); we get 


$$
\begin{aligned}
\frac{\partial K_{2}}{\partial t} & =\int\left\{\Lambda-d H-(\beta I+\alpha V) H+\rho I-\frac{H}{H^{*}}(\pi-d H-(\beta I+\alpha V) H+\rho I)+(\beta I+\alpha V) H\right. \\
-(a+\rho) I-\frac{I}{I^{*}}((\beta I+\alpha V) H+(a+\rho) I)+\frac{\alpha H^{*} V^{*}}{I^{*}}\left(d_{V} \Delta V+k I-u v+\frac{V^{*}}{V}\left(d_{V} \Delta V+k I-u v\right)\right) & \\
& \left.+\frac{\rho}{2(d+a)}\left(H-H^{*}+I-I^{*}\right) \cdot(\pi-d H+\rho I-(a+\rho) I)\right\} d x
\end{aligned}
$$

Then, by the same method using in the proof of Theorem 2 of in the paper of Zhang et al., we deduce that

$$
\begin{aligned}
\frac{\partial K_{2}}{\partial t} & =\int\left\{\left(d H^{*}-\beta I^{*} H^{*}-\rho I^{*}+\frac{d \rho H}{a+\rho}+\rho I\right)+\frac{\left(H-H^{*}\right)^{2}}{H H^{*}}-\frac{a \rho}{(d+a) H^{*}}\left(I-I^{*}\right)^{2}\right. \\
& \left.-\alpha H^{*} V^{*}\left(3-\frac{H^{*}}{H}+\frac{I^{*} V H}{H^{*} V^{*} I}-\frac{V^{*} I}{V I^{*}}\right)\right\} d x \\
& +\frac{\alpha d_{V} H^{*} V^{*}}{k I^{*}}\left(\int \Delta V d x-V^{*} \int \frac{\Delta V}{V} d x\right)
\end{aligned}
$$

Note that $\int \Delta V d x=0$ and $\int \frac{\Delta V}{V} d x=\int \frac{|\Delta V|^{2}}{V^{2}} d x$. Therefore, we have

$$
\begin{aligned}
\frac{\partial K_{2}}{\partial t} & =\int\left\{\left(d H^{*}-\beta I^{*} H^{*}-\rho I^{*}+\frac{d \rho H}{a+\rho}+\rho I\right)+\frac{\left(H-H^{*}\right)^{2}}{H H^{*}}-\frac{a \rho}{(d+a) H^{*}}\left(I-I^{*}\right)^{2}\right. \\
& \left.-\alpha H^{*} V^{*}\left(3-\frac{H^{*}}{H}+\frac{I^{*} V H}{H^{*} V^{*} I}-\frac{V^{*} I}{V I^{*}}\right)\right\} d x \\
& -\frac{\alpha d_{V} H^{*} V^{* 2}}{k I^{*}}\left(\int \frac{|\Delta V|^{2}}{V^{2}} d x\right)
\end{aligned}
$$

Since the arithmetic mean is greater than or equal to the geometric mean, it follows that

$$
3-\frac{H^{*}}{H}+\frac{I^{*} V H}{H^{*} V^{*} I}-\frac{V^{*} I}{V I^{*}} \leq 0 .
$$

Consequently if $R_{0}>1$ and $d H^{*}-\beta I^{*} H^{*}-\rho I^{*}>0$ then $\frac{\partial K_{2}}{\partial t} \leq 0$. Moreover, $\frac{\partial K_{2}}{\partial t}=0$ if and only $H=H^{*}, I=$ $I^{*}$ and $V=V^{*}$. Therefore, under above conditions if $M=\left\{E^{*}\right\}$ represents the largest compact invariant set in $\left\{(H, I, V): \frac{\partial K_{2}}{\partial t}=0\right\}$ and by the LaSalle's invariance principle [22] we deduce that $E^{*}$ is globally asymptotically stable whenever $R_{0}>1$ as the steady state $E^{*}$ exists whenever $R_{0}>1$.

Let remarks that the condition $d H^{*}-\beta I^{*} H^{*}-\rho I^{*} \geq 0$ is equivalent to

$$
1<R_{0} \leq 1+\frac{\left(\beta \pi+(a-\rho) d+\sqrt{(\beta \pi+(a-\rho) d)^{2}+4 a \rho d^{2}}\right)}{2 \rho d}=1+\delta
$$

\section{Numerical simulation}

In this section, we carry out some numerical simulations to illustrate the theoretical results obtained in the previous sections. For this purpose, we consider three sets of parameters values corresponding to the cases $R_{0}>1$. For the sake of simplicity of numerical simulations, we take one-dimensional spatial domain $\Omega=[0,50]$ and the values of the diffusion coefficient as $d_{V}=10$.

In the first test (Figures 1-3), we choose the following data set of system (3) $\Lambda=15, d=0.2, \beta=0.008, \alpha=$ $0.005, \rho=0.1, a=0.02, k=2$ and $u=1$. Using these values, ones get $R_{0}=1.125$ and $\delta=0.3583$ and we have $1<R_{0}<1+\delta$. In this case, system (3) has a epidemic equilibrium $E^{*}$ which is globally asymptotically stable (see Figures 1-3).

In Figures 4-6, we choose $\Lambda=20$ and do not change the other parameter values. By calculation, we have $R_{0}$ which satisfy Theorem 4; then the disease-free equilibrium is still present and the system (2) has a unique endemic equilibrium $E^{*}$. Therefore, by Theorem $4 E^{*}$ is globally asymptotically stable. Numerical simulation illustrates our result (see Figures 4-6). 


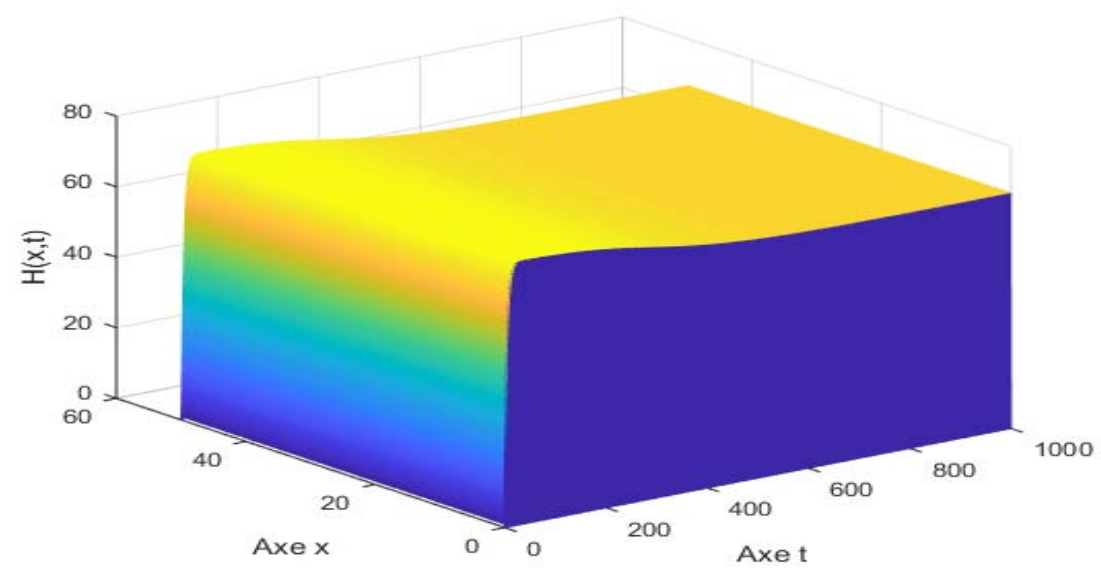

Figure 1. Concentrations of uninfected cells $\Lambda=15, d=0.2, \beta=0.008, \alpha=0.005, \rho=0.1, a=0.02, k=2$ and $u=1$.

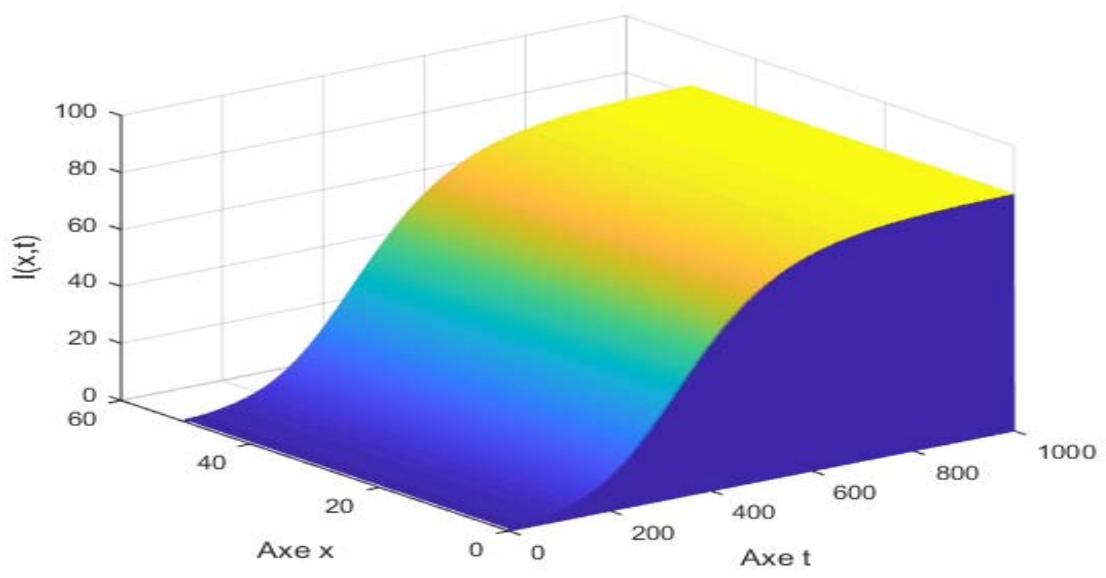

Figure 2. Concentrations of infected cells $\Lambda=15, d=0.2, \beta=0.008, \alpha=0.005, \rho=0.1, a=0.02, k=2$ and $u=1$.

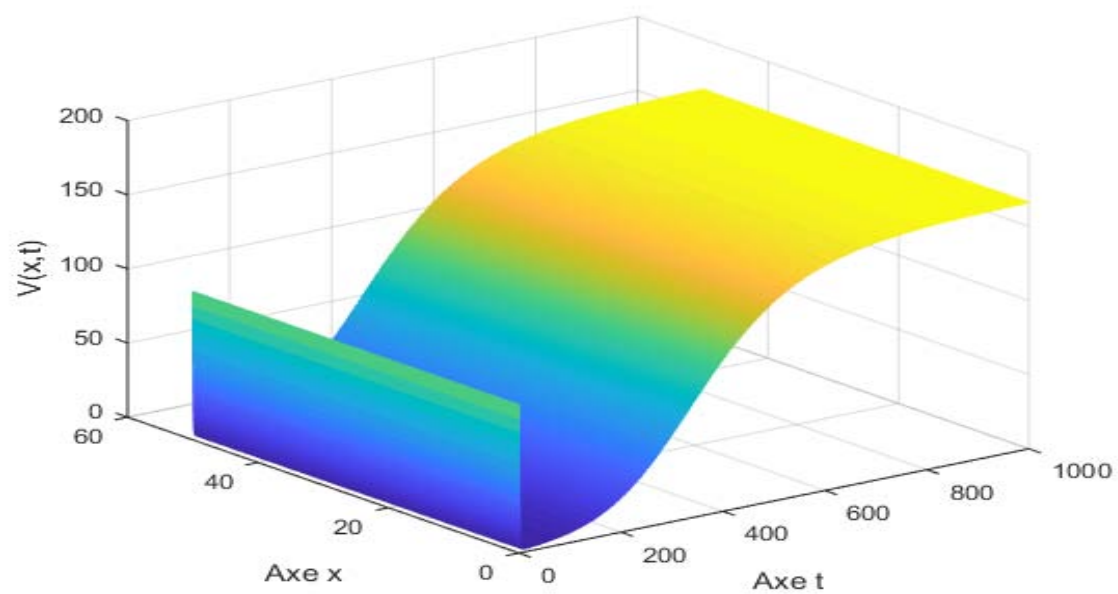

Figure 3. Concentrations of and free virus for $\Lambda=15, d=0.2, \beta=0.008, \alpha=0.005, \rho=0.1, a=0.02, k=2$ and $\boldsymbol{u}=\mathbf{1}$. 


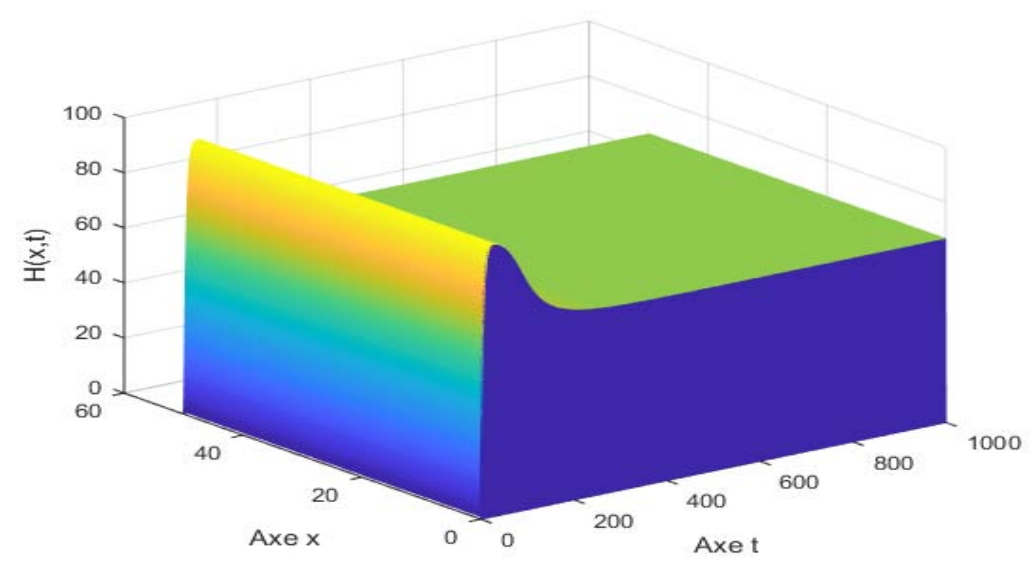

Figure 4. Concentrations of uninfected cells $\Lambda=20, d=0.2, \beta=0.008, \alpha=0.005, \rho=0.1, a=0.02, k=2$ and $\boldsymbol{u}=1$.

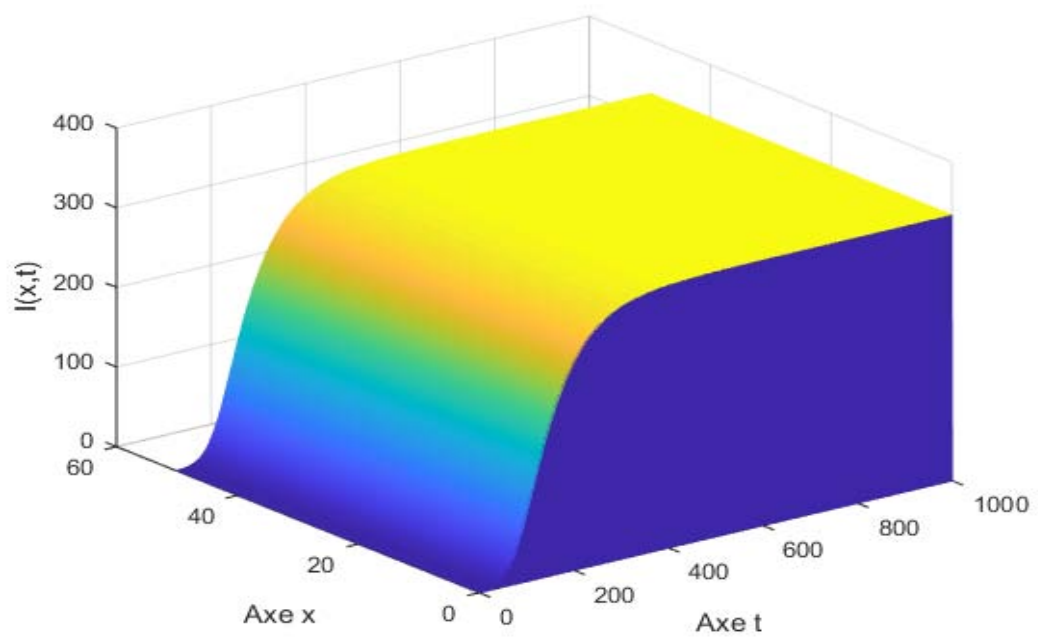

Figure 5. Concentrations of infected cells $\Lambda=20, d=0.2, \beta=0.008, \alpha=0.005, \rho=0.1, a=0.02, k=2$ and $u=1$.

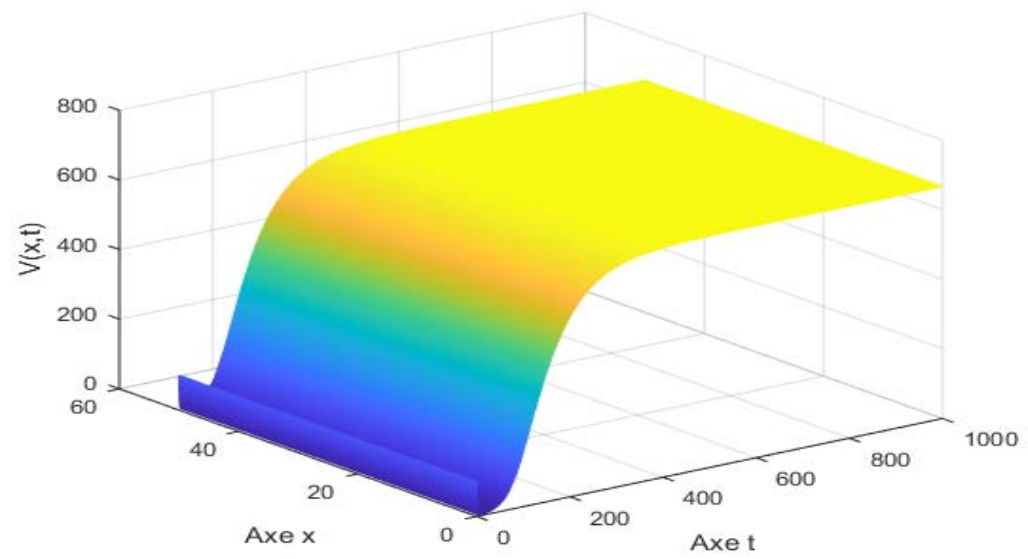

Figure 6. Concentrations of and free virus for $\Lambda=20, d=0.2, \beta=0.008, \alpha=0.005, \rho=0.1, a=0.02, k=2$ and $\boldsymbol{u}=1$. 


\section{References}

[1] Pasteur, L., Chamberland, C., and Roux, E. (1884). Physiologie exprimentale: nouvelle communication sur la rage (Experimental physiology: new communication on rabies). C. R. Acad. Sci., 98, 457-463.

[2] Chi, N. C., Vales, E., and Almeida, G. G. (2012). Analysis of a HBV model with diffusion and time delay. J. Appl. Math. Article ID 578561.

[3] Eikenberry, S., Hews, S., Nagy, J. D., and Kuang, Y. (2009). The dynamics of a delay model of hepatitis B virus infection with logistic hepatocyte growth. Math. Biosci. Eng., Vol. 6, No. 2, pp. 283-299.

[4] Hattaf, K. and Yousfi. (2013). Global stability for reaction-diffusion equations Comput. Math. Appl. Vol. 66, No. 8, pp. 1488-1497.

[5] Wang, J., Tian, X. (2013). Global stability of a delay differential equation of hepatitis B virus infection with immune response, Electron. J. Differ. Equ., Vol. 94, pp. 1-11.

[6] Zhang, Y., Xu, Z. (2014). Dynamics of a diffusive HBV model with delayed Beddington-DeAngelis response, Nonlinear Anal. Real World Appl., Vol. 15, 118-139.

[7] Perelson, A. S., Nelson, P. W. (1999). Mathematical analysis of HIV-1 dynamics in vivo. SIAM Rev., Vol. 41, No. 1, 3-44.

[8] Perelson, A. S., Neumann, A. U., Markowitz, M., Leonard, J. M., and Ho, D. D. (1996). HIV-1 dynamics in vivo: virion clearance rate, infected cell life-span, and viral generation time. Science, Vol. 271, No. 5255, 1582-1586.4.

[9] Nelson, P. W. and Perelson, A. S. (2002). Mathematical analysis of delay differential equation models of HIV-1 infection. Math. Biosci., Vol. 179, No. 1, 73-94.

[10] Spouge, J. L., Shrager, R. I., and Dimitrov, D. S. (1996). HIV-1 infection kinetics in tissue cultures, Math. Biosci., Vol. 138 No. 1, 1-22. https //:doi:org=10.1016/S0025-5564(96)00064- 8.

[11] Zhang, T., Meng, X., Zhang, T. (2015). Global dynamics of a virus dynamical model with cell-to-cell transmision and cure rate. Comput. Math. Methods Med. Article ID 758362.

[12] Zhang, T., Wang, J., Yuqing Li., Jiang, Z., and Han, X. (2020). Dynamics analysis of a delayed virus model with two different transmission methods and treatments. Adv. Differ. Equ., Vol. 2020, No 1, https://doi.org/10.1186/s13662-019-2438-0.

[13] Funk, G. A., Jansen, V. A. A., Bonhoeffer, S., and Killingback, T. (2005). Spatial models of virus-immune dynamics. J. Theor. Biol., Vol. 233, No. 2, 221-236.

[14] Manna, K. (2017). Global properties of a HBV infection model with HBV DNA-containing capsids and CTL immune response. Int. J. Appl. Comput. Math., Vol. 3, No. 3, 2323-2338.

[15] Pazy, A. (1983). Semigroups of Linear Operators and Applications to Partial Differential Equations. Vol. 44 of Applied Mathematical Sciences, Springer, New York, NY, USA.

[16] Protter, M. H. and Weinberger, H. F. (1967). Maximum Principles in Differential Equations. Prentice Hall, Englewood Cliffs.

[17] Alikakos, N. D. (1979). An application of the invariance principle to reaction-diffusion equations. Journal of Differential Equations. Vol. 33, No. 2, 201-225.

[18] Henry, D. (1993). Geometric Theory of Semilinear Parabolic Equations. Vol. 840 of Lecture Notes in Mathematics, Springer, Berlin, Germany.

[19] Diekmann, O., Heesterbeek, J. A. P., and Metz, J. A. (1990). On the definition and the computation of the basic reproduction ratio $R_{0}$ in models for infectious diseases in heterogeneous populations. Journal of Mathematical Biology, vol. 28, No. 4, 365-382.

[20] Van den Driessche, P. and Watmough, J. (2002). Reproduction numbers and sub-threshold endemic equilibria for compartmental models of disease transmission. Mathematical Biosciences, vol. 180, No. 1, 29-48.

[21] Routh, E. J., Clifford, W. K., Sturm, C., and Bocher, M. (1975). Stability of Motion. Taylor \& Francis, London, $U K$.

[22] La Salle, J. P. (1976). The Stability of Dynamica Systems. SIAM Publications, Philadelphia, Pa, USA. 